\title{
Aproximación al perfil de personalidad de adultos con trastorno del espectro autista mediante el MMPI-2: datos preliminares.
}

Towards a personality profile of adults with autism spectrum disorder using MMPI-2: preliminary data.

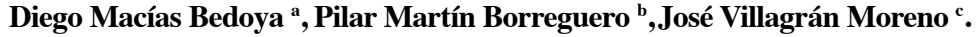 \\ ${ }^{a}$ Psicólogo clínico. Hospital de Jerez de la Frontera (Cádiz), España. ${ }^{b}$ Psicóloga clínica. Hospital de los \\ Morales. Córdoba, España. ${ }^{c}$ Psiquiatra. Coordinador. Hospital de Jerez de la Frontera (Cádiz), España.
}

Correspondencia: Diego Macías Bedoya (diego_macias_be@yahoo.es)

Recibido: 05/12/2013; aceptado con modificaciones: 24/05/2014

\begin{abstract}
RESUMEN: Introducción: La mayoría de estudios sobre personas con Trastornos del Espectro Autista (TEA) se centran en encontrar déficits en estas personas. La personalidad apenas ha recibido atención en este grupo de pacientes aunque con frecuencia una proporción de personas con TEA, aquellos que no tienen retraso mental, suelen confundirse con Trastornos de la Personalidad. El objetivo de este trabajo es conocer los rasgos de personalidad de un grupo de personas diagnosticadas con TEA y observar si existe un patrón de rasgos clínicos que se repita en los mismos.

Material y método: Se utilizó para la evaluación de la personalidad el Inventario Multifásico de Personalidad de Minnesota (MMPI-2). Para conocer el Cociente Intelectual se recurrió a la Escala de Inteligencia Wechsler para Adultos (WAIS-III). Igualmente se utilizaron las Escala de Observación para el Diagnóstico de Autismo (ADOS) y la Entrevista para el Diagnóstico del Autismo revisada (ADIR) para confirmar el diagnóstico de TEA. Se evaluaron 10 personas adultas con trastorno del espectro autista (TEA) de manera individualizada. Igualmente se utilizó a un grupo control de 10 personas sin diagnóstico de salud mental.
\end{abstract}

Resultados: Los resultados muestran puntuaciones elevadas en las escalas de validez L (mentira) y F (Incoherencia) y muy bajas en K (Factor Corrector). Igualmente se encuentran puntuaciones altas en las escalas clínicas 2 (Depresión) y 0 (Aislamiento Social) y en las escalas de contenido ANX (Ansiedad) SOD (Malestar Social) y OBS (Obsesividad) respecto al grupo control.

Conclusiones: Los resultados manifiestan un perfil de personalidad que incluye un solapamiento de síntomas notable con las características del Síndrome de Asperger. El MMPI-2 se revela como una potencial prueba de cribaje para dicho trastorno.

PALABRAS CLAVE (DECS): Síndrome de Asperger, Trastorno autístico, MMPI-2, determinación de la personalidad.
ABSTRACT: Introduction: The majority of studies into people with Autism Spectrum Disorders (ASD) focus on finding deficits in such people. Personality has scarcely received any attention in this group of patients, even though a proportion of people with ASD, those who have no mental delay, are often mistaken for people with Personality Disorders. The aim of this study is twofold: to identify the personality traits of a group of people diagnosed with ASD and also to determine whether there is a pattern of clinical traits in such a group.

Material and methods: The Minnesota Multiphasic Personality Inventory (MMPI-2)was used to measure personality. The Wechsler Adult Intelligence Scale (WAIS-III) was used to determine intelligence quotient. To confirm the ASD diagnosis the Autism Diagnostic Observation Schedule (ADOS) and the Autism Diagnostic InterviewRevised (ADIR) were used. Ten adults with Autism Spectrum Disorder (ASD) were individually evaluated. A control group of ten people with no mental health diagnosis was also used.

Results: Results show higher scores in ASP patients than controls in L (lie) and F (incoherence) validity scales, 2 (Depression) and 0 (Social isolation) clinical scales and ANX (Anxiety), SOD (Social Discomfort) and OBS (Obsessiveness) content scales, and lower scores in $\mathrm{K}$ (correction) factor.

Conclusions: Data obtained from this preliminary study show a personality profile similar to that of people with Asperger Syndrome. The MMPI-2 emerges as a test to be considered when diagnosing adults with this syndrome.

KEY WORDS (MESH): Asperger Syndrome, Autistic disorder, MMPI-2, personality assessment. 


\section{Introducción.}

El síndrome de Asperger se caracteriza por presentar un déficit cualitativo en la interacción social junto a la presencia de un repertorio restringido, estereotipado y repetitivo de actividades e intereses. Se diferencia del autismo en que para hacer este diagnóstico no deben existir déficits o retrasos del lenguaje o del desarrollo cognoscitivo (1). Lorna Wing fue la primera en utilizar el término Síndrome de Asperger en 1981 (2), si bien la primera persona que describió el cuadro fue Hans Asperger en 1944 en el artículo Die "Autistischen Psychopathen" in Kindesalter (3). El Dr. Asperger catalogó este grupo sindrómico como "psicopatía autista" y lo describió como un trastorno de la personalidad marcado principalmente por el aislamiento social (2).

La mayoría de los estudios sobre las personas con SA y otros trastornos del espectro autista (TEA) se ocupan, básicamente, de los déficits que muestran. Encontramos estudios que se centran en los problemas de comunicación (4-7), en la neurobiología (8), aspectos cognitivos (9), en las relaciones sociales (10), en los problemas de atención (11), y en los diferentes síntomas que componen el diagnóstico de TEA.

Por el contrario existen pocos estudios sobre los rasgos de personalidad de estos sujetos, a pesar de que con frecuencia se confunde el SA con trastornos diagnosticados en el Eje II, especialmente con el trastorno de la personalidad esquizoide, cuyas alteraciones conductuales presentan una sorprendente similitud con los síntomas asociados al SA (12). Así, cada vez más, se relacionan diferentes patologías mentales con trastornos de personalidad, encontrando una comorbilidad elevada en los mismos y superior a otros trastornos que se diagnostican en la clínica (13). Esta comorbilidad no es ajena a los TEA, como indican Anckarsäter y sus colaboradores en 2006, quienes señalan una alta prevalencia global de trastornos de la personalidad, especialmente con los clúster $\mathrm{A}$ y $\mathrm{C}$, en las personas con trastornos del espectro autista (14).

Igualmente el diagnóstico de SA presenta una alta comorbilidad con otros problemas mentales (15). Al mismo tiempo, el aspecto dimensional de los síntomas de las personas con SA no puede pasar desapercibido; así, los problemas para la interacción social o la falta de flexibilidad mental son comunes en la población general y se consideran rasgos de la personalidad o la forma de ser antes que manifestaciones psicopatológicas, sin que difieran cualitativamente de aspectos propios de cualquier individuo. La diferencia parece encontrarse en la expresión exagerada de algunas de estas características hasta el punto de interferir en la vida social (16). Si se acepta que los síntomas de las personas con SA pueden considerarse como rasgos acusados de la personalidad, y el hecho de la alta comorbilidad que conlleva este diagnóstico, la delimitación de un perfil característico de la personalidad que 
ORIGINALES Y REVISIONES

subyace en este trastorno, debe ser de ayuda en un mayor conocimiento y una más adecuada identificación del mismo y en la mejora de su tratamiento.

No hay muchos estudios que hayan evaluado la personalidad en el SA, y todos son posteriores al año 2000 lo que muestra que el interés en este tipo de investigación es reciente. En el primer estudio, del año 2001, Blackshaw, Kinderman, Hare y Hatton evaluaron, mediante una escala derivada de los ítems del MMPI, los síntomas de paranoia presentes en las personas con SA. Los autores encuentran que los participantes puntuaron significativamente más alto en la evaluación de la paranoia que el grupo control (17). Poco más tarde, en 2002, Soderstrom, Rastam y Gillberg utilizaron el Inventario de Temperamento y Carácter (TCI) de Cloninger, y evaluaron a 31 pacientes diagnosticados con SA. Encontraron que los participantes puntuaban significativamente más alto en la dimensión del temperamento Evitación del riesgo, y más bajo en dos dimensiones del carácter denominadas Autodirección y Cooperación. Además encontraron respuestas raras significativamente altas (18). En 2006, Anckarsäter y su equipo utilizaron de nuevo el TCI de Cloninger, obteniendo parecidos resultados, si bien utilizaron como grupo control pacientes con TDAH, y concluyeron que ambos grupos se asocian con configuraciones específicas de temperamento y un mayor riesgo de trastornos de la personalidad y déficit en la maduración del carácter (14).

En 2005, Elizabeth Austin, utiliza el Personality Mini-Markers y el Autism Spectrum Quotiem (AQ) para valorar a dos grupos de personas sin patología mental, los resultados muestran una relación entre mayores puntuaciones en el AQ y un patrón de personalidad con valores elevados en el factor Neuroticismo, baja puntuación en el factor Extraversión y en Amabilidad (19). Un año más tarde, Wakabayashi, Baron-Cohen y Wheelwright, utilizaron de nuevo el NEO-PI-R y el Autism Spectrum Questionnaire (AQ) con una muestra de estudiantes; encontraron que el AQ correlacionaba positivamente con el Neuroticismo y negativamente con la Extroversión y la Responsabilidad. Las diferencias encontradas, entre ambas investigaciones, en el factor Responsabilidad y Amabilidad las atribuyen los autores a las diferencias culturales entre las muestras utilizadas (Reino Unido en el estudio de Austin y Japón en el de Wakabayashi) así como a las diferencias entre las pruebas utilizadas (en el primero se utiliza el Personality Mini Marker y el estudio japonés utiliza el NEO-PI-R). Al mismo tiempo, señalan los autores que el modelo de cinco factores de personalidad (Big Five Personality) no predice la puntuación en el AQ ni siquiera en los casos en que las puntuaciones del AQ fueron muy elevadas. Más bien, sostienen, el trastorno autista sería una dimensión de la personalidad diferente a tener en cuenta. También sugieren que más importantes que los cinco factores de personalidad, lo son las diferentes facetas en que se dividen los mismos para obtener una aproximación más precisa a las personas con TEA (20).

En 2009, Sizoo realiza una investigación similar a la de Soderstrom y Anckarsäter en 2002 y 2006 respectivamente, utilizando de nuevo el TCI, en esta oca- 
sión en la población holandesa, y obteniendo resultados parecidos a los anteriores si bien encuentra que la variable historia de consumo de sustanciases importante; distinguiendo diferentes subgrupos en los TEA y TDAH en función de que haya existido o exista actualmente una historia de consumo (21). Otro estudio de personalidad sobre los TEA es el de Vuijk y colaboradores en 2012. En la investigación, de características similares a las anteriores, se utiliza el TCI de Cloninger y en lo referente al Temperamento, encuentran puntuaciones significativamente más bajas en Búsqueda de novedad, mayores en Evitación del riesgo y menores en Dependencia en la recompensa. Respecto al Carácter se obtienen puntuaciones más bajas en Autodirección y Cooperación (22).

Pero la investigación que ha supuesto el punto de partida de nuestro estudio ha sido la realizada por Ozonoff y sus colaboradores en 2005. Ozonoff y su equipo evaluaron a 20 personas adultas con TEA, todas con un CI superior a 70, y utilizaron como grupo control a alumnos universitarios. Les aplicaron el MMPI-2 y la autora encuentra que algunas de las escalas clínicas, como la Escala de Depresión (D) o Introversión Social (Si) están más elevadas en el grupo TEA que en el control. También encuentra más elevadas otras puntuaciones, como la escala de Malestar social (SOD) dentro de las escalas de Contenido; la escala de Represión (R), de Alcoholismo (MAC-R) y potencial adicción (APS) dentro de las escalas suplementarias y la de Introversión (INTR) dentro de las escalas PSY-5. Concluyen los autores, que encuentran similitudes importantes entre el cuadro clínico de los TEA y los correlatos de las escalas más elevadas respecto al grupo control, a saber, el malestar social y el aislamiento. Sugieren que el MMPI-2 recoge las características principales del fenotipo autista y puede ser una herramienta válida para evaluar la personalidad y psicopatología en esta población (23).

Por consiguiente, y a tenor de lo expuesto, la evaluación de las características de la personalidad de los sujetos con TEA, incluido el SA, se ve justificada por la escasez de investigaciones al respecto, por la utilidad de los hallazgos apuntados en ellas para un mejor conocimiento de estos trastornos y porque la consolidación de una relación más o menos reiterada entre el diagnóstico de TEA y los resultados de una prueba de tipo estandarizado puede ayudar a mejorar el diagnóstico diferencial y evitar la confusión nosológica. El presente estudio pretende aproximarse al conocimiento de las particularidades existentes en la personalidad de las personas con Síndrome de Asperger(SA). 
ORIGINALES Y REVISIONES

\section{Método.}

\subsection{Participantes.}

Como análisis preliminar, parte de un estudio más amplio que constituye el tema de la tesis doctoral del primero de los autores (DM), se evaluaron 10 personas adultas con Trastorno del Espectro Autista (TEA), 9 de ellas con diagnóstico de Síndrome de Asperger(SA) y una con diagnóstico de TGD no especificado, siguiendo los criterios CIE-10.Se utilizaron como grupo control otras 10 personas, adultos, profesionales pertenecientes a la Unidad de Salud Mental Infanto-Juvenil de Córdoba del Servicio Andaluz de Salud. A todos ellos se les administró de forma individualizada el Inventario de Personalidad Multifactorial de Minnesota (MMPI2). Ninguna de las pruebas fue invalidada por lo que todos los protocolos fueron considerados correctos. Todos los participantes, tanto en el grupo control como el grupo de personas con TEA, tenían un Cociente Intelectual superior a 70 evaluado con la escala de Weschler WAIS-III, no habiendo ningún caso de retraso mental en los evaluados. Al mismo tiempo, se descartó la existencia de síntomas de la esfera autista en el grupo control (siguiendo los criterios de la CIE-10). También se descartó la existencia de otro tipo de trastorno mental en ambos grupos, por lo que ninguno de ellos recibía ningún tipo de tratamiento de salud mental.

\subsection{Pruebas de evaluación.}

Inventario Multifásico de Personalidad de Minnesota (MMPI-2) (24). Consiste en un inventario autoaplicado de 567 ítems de respuesta verdadero-falso. Arroja un total de 6 escalas de validez, 10 escalas clínicas básicas y 15 escalas de contenido, junto a otras escalas que también pueden ser consideradas. Esta prueba fue realizada de forma individualizada por cada uno de los participantes del estudio. En este estudio solo se consideraron las escalas clínicas, de validez y de contenido por ser estas las más utilizadas en la clínica.

Escala de Observación para el Diagnóstico de Autismo (ADOS) (25). Es una prueba estandarizada y semi-estructurada de la comunicación, la interacción social y el juego o el uso imaginativo de materiales para sujetos con sospecha de TEA. Todos los participantes de la investigación diagnosticados como TEA fueron evaluados mediante esta prueba diagnóstica superando en todos los casos los puntos de corte para el diagnóstico.

Entrevista para el Diagnóstico del Autismo revisada (ADIR) (26). Se trata de una entrevista clínica que permite evaluar sujetos con sospechas de algún TEA. La entrevista se realiza con familiares cercanos a la persona afectada y se trata de conocer conductas que están presentes o no a lo largo del desarrollo del sujeto. Igual que en el caso anterior todas las personas diagnosticadas como SA fueron evaluadas con esta prueba. 
Escala de Inteligencia de Wechsler para Adultos (WAIS-III) (27). Se trata probablemente de la prueba de inteligencia más utilizada. Consta de 14 subtests y permite obtener tres tipos de CI (verbal, manipulativo y total) y cuatro índices específicos. Todos los sujetos con trastorno del desarrollo fueron evaluados mediante esta prueba, situándose en todos los casos por encima del retraso mental.

\subsection{Pruebas estadísticas.}

Se utilizó la prueba de Kolgomorov-Smirnov para comprobar si los valores se ajustan a la normalidad. Para un intervalo de confianza del 95\%, (p>0,05)se concluyó que la distribución de los valores es normal. Posteriormente se aplicó la prueba T de Student para comprobar si existen diferencias entre las medias obtenidas por el grupo TEA y el grupo control. Finalmente se calcularon las correlaciones de Pearson para comparar la edad y los resultados del ADI-R con cada una de las escalas del MMPI-2 valoradas. Para realizar estos cálculos estadísticos se utilizó el paquete informático IBM SPSS Statistics 20.

\section{Resultados.}

$\mathrm{Al}$ ser la muestra de este estudio preliminar de tamaño reducido, se ha considerado en la interpretación de los resultados no solo la significación estadística sino también los efectos de tamaño para categorizar los datos obtenidos.

Respecto a las variables recogidas de los grupos, no se encuentran diferencias entre ellos en cuánto a género ni existencia de retraso mental (Tabla 1). Sí se hallaron diferencias respecto a la edad del grupo, siendo más alta en el grupo control que en el grupo experimental.

Resumen de los datos demográficos recogidos.

\begin{tabular}{|c|c|c|c|c|}
\hline & \multicolumn{3}{|c|}{ Grupo TEA } & Grupo Control \\
\hline Edad & \multicolumn{3}{|c|}{$21,3(3,97)$} & $35(7,76)$ \\
\hline Género \% varones & \multicolumn{3}{|c|}{100} & 100 \\
\hline ADOS & \multicolumn{3}{|c|}{ Todos por encima del algoritmo diagnóstico } & N. A. \\
\hline $\begin{array}{l}\text { ADI-R (Comunicación-social-patrones } \\
\text { repetitivos) }\end{array}$ & $8(6-12)$ & $14(10-18)$ & $3(1-4)$ & N. A. \\
\hline WAIS-III & \multicolumn{3}{|c|}{$>70$} & $>70$ \\
\hline
\end{tabular}


ORIGINALES Y REVISIONES

Siguiendo a Cohen (28), categorizamos los efectos obtenidos como; pequeños de 0,20 a 0,49 ; efecto medio desde 0,50 hasta 0,79 ; y efecto grande los que están por encima de 0,80 .

En referencia a los resultados obtenidos en el MMPI-2; una primera aproximación permite observar, una vez obtenidas las puntuaciones $\mathrm{T}$ de cada uno de los grupos, que las puntuaciones del grupo TEA se encuentran, en la mayoría de las escalas, por encima de la media de la prueba. Por el contrario, las del grupo control, se encuentran por debajo de la media esperada. Asimismo, hallamos desviaciones típicas elevadas en el grupo TEA, frente a las del grupo control, en éste último en la mayoría de los casos está por debajo de 10. Los valores obtenidos en el grupo control muestran un grupo homogéneo en cuanto a las puntuaciones obtenidas y con unas puntuaciones muy bajas que difieren de la esperada para una población normal (ver gráficos 1 y 2).

Gráfico 1:

Comparación resultados grupo TEA y Control en las Escalas Clínicas.

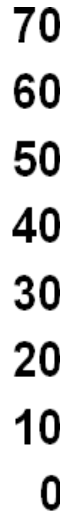

70
60
50
40
30
20
10
0

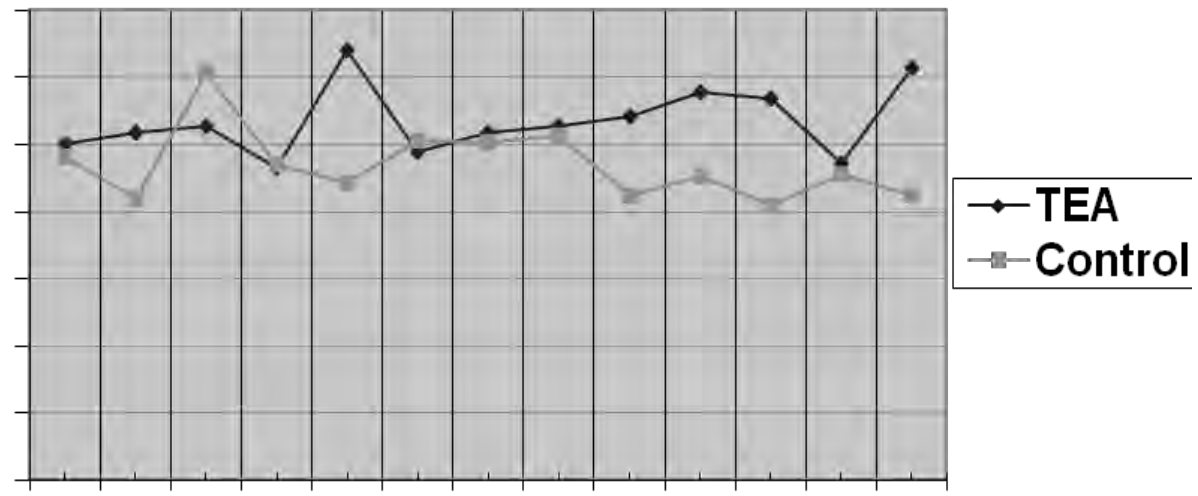

$\begin{array}{lllllllllllll}\text { L } & \mathbf{F} & \mathrm{K} & \mathrm{Hs} & \mathbf{D} & \mathrm{Hy} & \mathrm{Pd} & \mathrm{Mf} & \mathrm{Pa} & \mathrm{Pt} & \mathrm{Sc} & \mathrm{Ma} & \mathrm{Si}\end{array}$

Gráfico 2:

Comparación resultados grupo TEA y Control en las Escalas de Contenido.

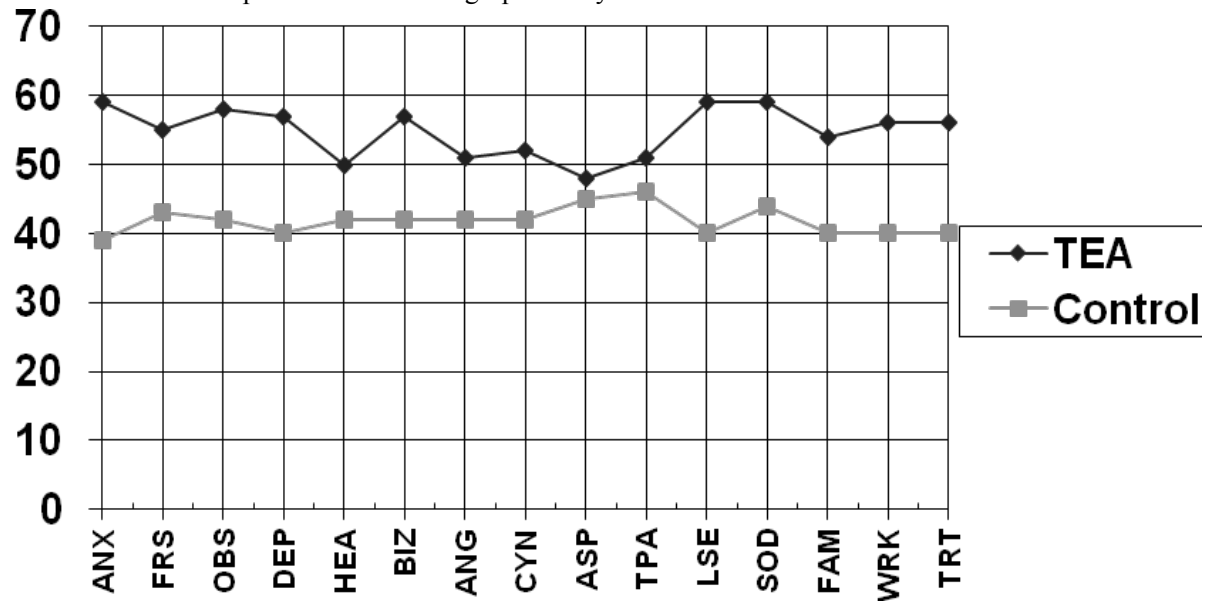


Considerando los resultados de las diferentes escalas, un primer grupo de hallazgos corresponden a las Escalas de Validez: L, F y K. El grupo TEA obtiene puntuaciones medias superiores en L $(50)$ y $\mathrm{F}(51,8)$ frente al grupo control $(48,2$ y 42 respectivamente), no así en $\mathrm{K}$ en que se invierte la tendencia frente al grupo control (52,8 y 60,7). Frente a la muestra del MMPI-2, el grupo TEA se encuentra igualado en la escala $\mathrm{L}$ y por encima en las escalas $\mathrm{F}$ y $\mathrm{K}$.

En lo concerniente a las Escalas Clínicas; el grupo de personas con TEA obtiene valores superiores al grupo control en todos los casos, excepto en la escala 3 (Hy) en que el grupo control muestra medidas superiores (50,7 frente a 49), y en la escala $1(\mathrm{Hs})$, en que no se obtienen medias diferentes estadísticamente $(46,5 \mathrm{y}$ $47,1)$. En las demás escalas, las mayores diferencias las encontramos en las escalas2 (D) (64 y 44,4), 0 (Si) (61,3 y 42,5), 8 (Sc) $(56,8$ frente a 41), 7 (Pt) $(57,8$ y $45,3)$ y $6(\mathrm{~Pa})(54,1$ y 42,3$)$, todas con diferencias grandes. Si comparamos el grupo TEA con la muestra del MMPI-2, encontramos un patrón de personalidad similar pero con diferencias menores, así pues, la escala 2 (D) sigue siendo la más alta (64 frente a 50), seguida en este caso de $0(\mathrm{Si})$ (61,3 y 50), ambas, siguiendo a Cohen, con diferencias grandes, le seguirían con diferencias medias, la escala $7(\mathrm{Pt})(57,8$ y 50) y $8(\mathrm{Sc})(56,8$ frente a 50). No encontraríamos una diferencia relevante en la escala 6 (Pa) $(54,1$ y 50$)$, (tabla 2$)$.

Tabla 2.

Resultados obtenidos en las escalas del MMPI-2

\begin{tabular}{|c|c|c|c|}
\hline Escalas MMPI-2 & Grupo TEA & Grupo Control & Tamaño del efecto, $d$ \\
\hline $\mathbf{L}$ & $50(6,43)$ & $48(7,31)$ & 0,29 \\
\hline $\mathbf{F}$ & $52(12)$ & $42(3,19)$ & 1,32 \\
\hline $\mathbf{K}$ & $53(2,2)$ & $61(8,42)$ & 1,50 \\
\hline $1(\mathrm{Hs})$ & $47(9,22)$ & $47(6,47)$ & 0,01 \\
\hline 2 (D) & $64(13,06)$ & $44(3,74)$ & 2,38 \\
\hline 3 (Нy) & $49(7,96)$ & $51(6,68)$ & 0,27 \\
\hline $4(\mathrm{Pd})$ & $52(12,25)$ & $50(4,81)$ & 0,23 \\
\hline 5 (Mf) & $53(11,16)$ & $51(9,14)$ & 0,20 \\
\hline $6(\mathrm{~Pa})$ & $54(16,27)$ & $42(5,87)$ & 0,90 \\
\hline $7(\mathrm{Pt})$ & $58(14,72)$ & $45(7,26)$ & 1,18 \\
\hline $8(\mathrm{Sc})$ & $57(15,26)$ & $41(3,86)$ & 1,67 \\
\hline 9 (Ma) & $47(8,77)$ & $46(7,74)$ & 0,12 \\
\hline
\end{tabular}




\begin{tabular}{llll}
\hline $0($ Si $)$ & $61(14,48)$ & $43(5,64)$ & 1,79 \\
\hline ANX & $59(11,35)$ & $39(3,19)$ & 2,75 \\
\hline FRS & $55(13,24)$ & $43(6,96)$ & 1,188 \\
\hline OBS & $58(11,55)$ & $42(7,65)$ & 1,67 \\
DEP & $57(16,27)$ & $40(4)$ & 1,67 \\
\hline HEA & $50(6,98)$ & $42(3,03)$ & 1,6 \\
\hline BIZ & $57(12,48)$ & $42(3,01)$ & 1,93 \\
\hline ANG & $51(11,18)$ & $44(3,81)$ & 1 \\
\hline CYN & $52(7,56)$ & $42(5,41)$ & 1,54 \\
\hline ASP & $48(5,7)$ & $45(6)$ & 0,51 \\
TPA & $51(7,42)$ & $46(3,27)$ & 0,94 \\
LSE & $59(17)$ & $40(3,43)$ & 1,87 \\
SOD & $59(12,63)$ & $44(6,17)$ & 1,6 \\
\hline FAM & $54(9,37)$ & $40(4,48)$ & 2,02 \\
WRK & $56(14,9)$ & $40(4,54)$ & 2,01 \\
\hline TRT & $56(11,81)$ & $40(3,93)$ & 2,03 \\
\hline
\end{tabular}

Tabla 2 (cont). Resultados obtenidos en las escalas del MMPI-2

Finalmente, en las Escalas de Contenido, en todas las ocasiones el grupo TEA muestra puntuaciones superiores a las del grupo control, además este grupo muestra puntuaciones T muy bajas, excepto en la escala TPA (obtiene una media de 46), con valores medios iguales o inferiores a 45. Este motivo hace que los efectos de tamaño arrojen valores muy elevados, así en comparación con el grupo control, los efectos podemos clasificarlos como grandes en todas las escalas de contenido excepto en la escala ASP. Al comparar el grupo TEA con la muestra del MMPI-2 los resultados sí difieren de forma importante respecto a los obtenidos en comparación con el grupo control; así, los resultados nos muestran una sola diferencia por encima de 0,80 y que podemos catalogar como grande, la correspondiente a la escala $\operatorname{ANX}(58,5)$. Dentro de los efectos medios encontramos, por orden de tamaño de diferencias: SOD (muy cercano al efecto grande con 0,79$)$, OBS $(57,6)$, LSE $(58,5)$, BIZ $(56,5)$, TRT $(56,2)$ y DEP $(56,9)$.

Correlaciones de Pearson.

Se calcularon las correlaciones de Pearson comparando la edad y los resultados del ADI-R con cada una de las escalas del MMPI-2 valoradas. Considerando 
nuevamente los efectos del tamaño, según Cohen, en el caso de Pearson, se suponen efectos medianos a partir de 0,30 y hasta 0,49 , mientras se denominan efectos grandes a partir de 0,50.El resultado más destacable es el obtenido al comparar la edad del grupo TEA con las puntuaciones de la prueba ADIR, se obtiene una correlación negativa $(-0,345)$ dentro de los efectos medios, que sugiere que a mayor edad el grado de afectación es menor tanto en la puntuación completa del ADIR como en cada uno de las dimensiones (Comunicación, social y hábitos repetitivos) que evalúa.

\section{Discusión.}

La investigación realizada pretende ser una primera aproximación al perfil de personalidad de las personas con trastornos del espectro autista. El carácter exploratorio de esta investigación y el reducido tamaño de la muestra, hacen que los resultados obtenidos deban ser interpretados con prudencia y siempre considerando la dificultad que supone generalizar las conclusiones extraídas de los mismos.

Entre los hallazgos obtenidos encontramos un grupo de personas con TEA con una edad inferior a la del grupo control, pero igualados en cuanto a género e inteligencia. Respecto a los resultados de la investigación de Ozonoff, descubrimos que los valores obtenidos por la muestra TEA de nuestra investigación son similares a los obtenidos por la autora americana, mientras los valores del grupo control son muy inferiores a los que aparecen en su investigación, especialmente en las puntuaciones de las escalas de Contenido.

En relación a la comparación en las escalas del MMPI-2, en las escalas de validez L, F y K obtenemos resultados similares a los de Ozonoff, con valores más alto en el grupo TEA en L y $\mathrm{F}$ e inferiores en $\mathrm{K}$. Altos valores en $\mathrm{L}$ se relacionan con personas defensivas, convencionales, rígidas, que responden al azar o que niegan sus problemas, valores muy elevados informan también de posible ingenuidad en sus respuestas (29). Algunas de estas características describen a las personas del espectro autista: pensamos que no son las respuestas al azar ni la negación las que justifican las altas puntuaciones, sino que las características de rigidez e ingenuidad, descritas con frecuencia en relación a estas personas (30-31, 5) pueden indicar esta elevación en la escala L. Por otro lado, la otra escala de validez, la escala F, se refiere a la incoherencia en las respuestas; valores elevados indican, falta de cooperación, contestar de forma azarosa, problemas de lectura, exageración de síntomas, pero también problemas emocionales significativos. Esta última idea debe ir corroborada por las elevaciones de las puntuaciones del perfil clínico, que indicará la presencia o no de estas dificultades. Nuevamente la existencia de un perfil 
ORIGINALES Y REVISIONES

clínico alterado nos indica que la elevación de la escala $\mathrm{F}$ puede estar justificada por las dificultades emocionales que se manifiestan en las altas puntuaciones de las diferentes escalas del perfil clínico.

Sobre las escalas Clínicas, encontramos un perfil con valores muy elevados en 2 y 0 , así como en 7, 8 y 6 . El perfil 2-0 es reflejo de rasgos de carácter depresivo, con valores personales rígidos e introversión social elevada con preferencia por estar solo y falta de asertividad social. Las otras dimensiones elevadas informan de una probable presencia de rituales compulsivos, obsesiones y síntomas similares al trastorno obsesivo compulsivo, acompañado de alienación social y emocional, así como sensibilidad y suspicacia frente a los demás. Este patrón coincide con la idea que actualmente se tiene de lo que es una persona con SA así como de los síntomas descritos en los manuales diagnósticos. Además, son resultados parecidos a los obtenidos por Ozonoff. Así, también ella encuentra un perfil 2-0, y puntuaciones elevadas en 8. Por el contrario, en su investigación, encuentra puntuaciones elevadas en 9 que no aparecen en nuestro estudio, y no encuentra valores elevados en $7 \mathrm{y}$ 6. La presencia de síntomas de depresión en adultos con SA ha sido con frecuencia indicada en numerosos estudios sobre esta población $(5,15,31-34)$, a pesar de que ninguno de las personas del grupo TEA tenía un diagnóstico adicional de depresión ni recibía tratamiento para este trastorno. Así, parece que la tristeza y el desánimo forman parte de las manifestaciones de las personas con SA sin que sea necesario que cumplan los criterios diagnósticos de un trastorno del ánimo depresivo propiamente. Este hallazgo se refrenda por las altas puntuaciones obtenidas en las escalas de Contenido DEP y LSE que caracterizan a individuos con pensamientos depresivos, escaso interés por sus vidas, tristeza y desesperanza, así como baja autoestima y actitudes negativas sobre sí mismo. La introversión social es uno de los síntomas nucleares de las personas con TEA y que se encuentran en los criterios diagnósticos del SA. En las escalas de Contenido se encuentra reflejada en las altas puntuaciones obtenidas en la escala SOD que define a personas que se sienten intranquilas en situaciones sociales y que prefieren estar solas definiéndose como tímidos y con poco interés en los actos sociales (29).

La presencia de rituales, preocupaciones obsesivas y miedos, reflejada en la escala Clínica 7, aparece de nuevo refrendada en las escalas de Contenido mediante las altas puntuaciones obtenidas en OBS, que indican problemas para tomar decisiones, dificultades para hacer cambios e interés por coleccionar cosas y en ANX, que indica, entre otros síntomas, ansiedad general, preocupaciones y problemas para tomar decisiones (29). De nuevo estos rasgos coinciden, como en los anteriores casos, con las dificultades características de las personas con SA.

Las altas puntuaciones en la escala Clínica 8 vienen acompañadas por altas puntuaciones en las escala de Contenido BIZ que hace referencia a pensamientos de tipo extravagante, posible existencia de alucinaciones, así como ideación de tipo para- 
noide, síntoma éste que se vería refrendado por las altas puntuaciones obtenidas en la escala Clínica 6. En general estos síntomas no son frecuentes ni definitorios de las personas con SA; la escala 8 hace también referencia a alienación social y emocional, así como a reserva social, igualmente la desconfianza y suspicacia frente a los demás puede estar justificada por los problemas que les suscitan, a los afectados con TEA, el contacto con otras personas. La explicación sobre los altos valores en la escala de Contenido BIZ es más difícil de vislumbrar; esta escala se relaciona con la escala Clínica 8 y hace referencia a rasgos de personalidad de tipo psicótico (29).

En otra investigación en la que se utilizó el MMPI-2 (17), los autores evaluaron posibles síntomas de paranoia en personas con TEA mediante la escala 6 del MMPI-2, descubriendo puntuaciones elevadas en el grupo TEA frente al grupo control. En nuestro estudio encontramos puntuaciones elevadas en el grupo TEA en la escala $6(\mathrm{~Pa})$, frente al grupo control, si bien estas diferencias no aparecen al utilizar como grupo de comparación la muestra del MMPI-2. Sin embargo, un $40 \%$ de la muestra TEA presentó valores en esta escala por encima de 65 en las puntuaciones T del MMPI. La escala 6 valora tres dimensiones dentro de los síntomas de la paranoia: ideas persecutorias, hipersensibilidad e ingenuidad. De estas tres dimensiones, la hipersensibilidad hace referencia a personas que se sienten tensos con frecuencia y que se ven afectados con facilidad en las relaciones personales y que manifiestan sentimientos de soledad e incomprensión; la ingenuidad, por otro lado, valora la sinceridad, la existencia de normas morales y la falta de impulsos hostiles negativos (29). Creemos que son estas dos dimensiones, las que pueden justificar los altos valores de la escala 6 , más que las ideas persecutorias en sí, que caracterizan a los trastornos de tipo paranoide.

Respecto a las investigaciones realizadas con el TCI de Cloninger, coinciden en los resultados obtenidos, a saber; obtienen puntuaciones más altas en el grupo TEA en Evitación del Riesgo, una dimensión que se subdivide en varias escalas: Preocupación y pesimismo, Temor a la incertidumbre, Timidez social y Fatigabilidad; más bajas en Búsqueda de la novedad, que a su vez está formada por las escalas Excitabilidad exploratoria, Impulsividad, Prodigalidad y Desorden (infracciones) y puntuaciones bajas también en Dependencia de la recompensa que incluye Sentimentalidad, Apertura a la comunicación, Apego y Dependencia. Estos resultados presentan un fenotipo de las personas con TEA en el que destacan las peculiaridades de pesimismo, temor ante actividades nuevas, distanciamiento social y fatigabilidad, poco interés en la búsqueda de estímulos novedosos, reflexivos y normativos e interesados en mantener rutinas o actividades que les resultan familiares; poco interesados en comunicarse socialmente y mostrar sus sentimientos, así como escaso apego por las personas (22). Si bien es cierto que resulta difícil comparar los resultados de diferentes pruebas de personalidad, también es cierto 
ORIGINALES Y REVISIONES

que la descripción realizada por el grupo de Vuijk sobre las personas con TEA es muy cercana a la que expone el perfil encontrado con el MMPI-2.

Respecto a los resultados obtenidos con el modelo de Cinco Factores, el patrón configurado por alto Neuroticismo, baja Extraversión y baja Amabilidad (en el caso de Austin) o baja Responsabilidad (en el caso de Wakabayashi), resulta poco preciso para aproximarnos a un perfil de personalidad concreto; pensamos, como señalan los autores de la investigación de Wakabayashi y sus colaboradores (20), que el modelo de Cinco Factores no se ajusta tanto al perfil buscado y que es necesario analizar mayor número de dimensiones para conseguir un perfil más ajustado. Estamos de acuerdo en que pueden ser más útiles las dimensiones en que se dividen cada uno de los factores de personalidad que los propios grandes factores.

En definitiva, el perfil obtenido mediante el MMPI-2 coincide en gran parte con las investigaciones precedentes a la nuestra y muestra una gran similitud con la descripción clínica de las personas con TEA. El aislamiento social y la presencia de rituales y obsesiones aparecen como criterios diagnósticos en el SA desde los rasgos clínicos de la psicopatía autística de Hans Asperger en 1.944, pasando por las características clínicas de Lorna Wing en 1.981, las pautas de diagnóstico de Gillberg, Szatmari y Tantam a fines de los 80 e inicios de los 90, hasta los criterios diagnósticos actuales de la APA (35) y la OMS $(1,12)$. Los síntomas de depresión son, por otro lado, una tónica casi constante en la adolescencia y adultez de las personas con SA como se señaló anteriormente. Coincidimos con Ozonoff (23) en que el MMPI-2 captura con precisión el fenotipo de las personas con TEA y podría constituir en el futuro una herramienta válida para valorar la personalidad y psicopatología en esta población.

Nuestra investigación adolece de importantes limitaciones, algunas de las cuáles se han señalado ya, pero creemos que conviene recordarlas en la discusión final; el tamaño de la muestra constituye el principal problema, tanto del grupo clínico como del grupo control. Igualmente, el grupo control presenta características que le hacen alejarse de los resultados esperados para la prueba de personalidad utilizada (sus integrantes forman parte del personal perteneciente a la Unidad de Salud Mental Infanto-Juvenil de Córdoba lo que puede constituir un sesgo). Además, no se ha utilizado un grupo control clínico con el que comparar el grupo TEA y también el grupo control. También ha habido diferentes variables que no se han tenido en cuenta en la recogida de datos como son el nivel de estudios de los participantes, el cociente intelectual, tanto el resultado total como la posible diferencia de perfiles verbales o manipulativos no fue valorado psicométricamente, tampoco se realizó una evaluación de posible patología en el grupo control. Estas condiciones hacen que sea necesario realizar una oportuna réplica que utilice mayor número de participantes y evite las limitaciones de este estudio.

El diagnóstico de las personas con TEA, especialmente cuando no existe 
retraso mental, presentan habilidades verbales y no hay un marcado aislamiento social, como en el caso de las personas con SA, continúa siendo con demasiada frecuencia tardío. Incluso en las etapas superiores de la adolescencia y la adultez encontramos en los servicios de Salud Mental personas que cumpliendo los criterios diagnósticos de este síndrome reciben diferentes etiquetas diagnósticas erróneas. Así, todavía existe gran número de individuos adultos inteligentes con dificultades severas para interactuar y comunicarse con otros que no han recibido un diagnóstico específico y por tanto no han obtenido una ayuda efectiva (12). Al mismo tiempo es frecuente que hayan realizado diferentes pruebas en forma de test, cuestionarios, inventarios que difícilmente han conducido a un diagnóstico de TEA. Aquí radica la importancia de obtener un perfil característico con una prueba de screening, no específica de las personas con TEA, como es el MMPI-2, utilizada con frecuencia en la práctica clínica de la salud mental, que nos permita detectar el fenotipo básico de estas personas y hacernos sospechar de la posible presencia de un trastorno de la esfera autista. Acercarnos a este objetivo puede permitirnos en el futuro mejorar los problemas actuales para el diagnóstico de adultos con síntomas autistas leves.

\section{BIBLIOGRAFÍA}

(1) Organización Mundial de la Salud. OMS. Clasificación de los trastornos mentales y del comportamiento CIE-10.Madrid: Panamericana, 2006.

(2) Wing L. Asperger syndrome: a clinical account. Psychol Med, 1981; 11:115-29.

(3) Asperger H. Die "AutistischenPsychopathen" in Kindesalter. ArchPsychiatrNervenkr, 1044;117:76-136.

(4) Rodríguez Muñoz FJ.Comunicación, autismo y síndrome de asperger. Hacia un estado de la cuestión desde el punto de vista bibliográfico. Revelectronestudfilológ, 2007;13.

(5) Attwood T. The Complete Guide to Asperger's Syndrome. London: Jessica Kingsley Publishers, 2007.

(6) Saulnier C, Klin A. Brief Report: Social and Communication Abilities and Disabilities in Higher Functioning Individuals with Autism and Asperger Syndrome, JAutism DevDisord, 2007;37:788-93.

(7) Korpilahti P, Jansson-Verkasalo E, Mattila M, Kuusikko S, Suominen K, Rytky S, Pauls D, Moilanen I. Processing of Affective Speech Prosody is Impaired in Asperger Syndrome. JAutismDevDisord, 2007;37: 1539-49.

(8) Alonso JR, Gómez C, Valero J, Recio JS, Baltanás FC, Weruaga E. Investigación en neurobiología del síndrome de asperger. Últimos resultados en investigación. Actas Primeras Jornadas sobre Síndrome de Asperger, Sevilla, 2005. 2000;1:34-44.

(9) Artigas A. Aspectos neurocognitivos del síndrome de asperger. Rev NeurolClín,

(10) Rao P,Beidel D, Murray M.Social skills interventions for children with Asperger's 
ORIGINALES Y REVISIONES

Syndrome or High-Functioning Autism: A review and recommendations. J Autism DevDisord, 2008;38:353-61.

(11) Ehlers S, Nydén A, GillbergCh, Sandberg A, Dahlgren S, Hjelmquist E, Odén A. Asperger Syndrome, Autism and Attention Disorders: A comparative study of the cognitive profiles of 120 children. JChildPsycholPsychiatr, 2006;38 (2):207-17.

(12) Martín Borreguero P. El síndrome de Asperger: ¿Excentricidad o discapacidad social? Madrid: Alianza, 2008.

(13) PailhezVindual G, Palomo Nicolau A.Multiplicidad diagnóstica en los trastornos de la personalidad, Psiquiatrbiol, 2007; 14 (3) 92-7.

(14) Anckarsäter H, Stahlberg O, Larson T, Hakansson C, Jutblad S, Niklasson L, Nydén A, Wentz E,Westergren S, Cloninger R, GillbergCh, Rastam M. The impact of ADHD and autism spectrum disorders on temperament, character and personality development.Am J Psychiatr, 2006;163:1239-44.

(15) Lugnegårda T, Hallerbäckc MU,Gillberg Ch.Psychiatric comorbidity in young adults with a clinical diagnosis of Asperger syndrome. DevDisabil, 2011;32:1910-17.

(16) Martos J, Freire S, Ayuda R, González A, Llorente M, Martínez C, Artigas J.Un acercamiento al Síndrome de Asperger: una guía teórica y práctica.Valencia: Imserso, 2004.

(17) Blackshaw AJ, Kinderman P, Hare DJ, Hatton C.Theory of mind, causal attribution and paranoia in Asperger syndrome. Autism 2001;5:147-63.

(18) Soderstrom H, Rastam M, Gillberg C.Temperament and character in adults with Asperger Syndrome. Autism 2002;6:287-97.

(19) Austin E. Personality correlates of the broader autism phenotype as assessed by the Autism Spectrum Quotient (AQ). Personal Individ Differ, 2005;38 (2):451-60.

(20) Wakabayashi A, Baron-Cohen S, Wheelwright S.Are autistic traits an independent personality dimension? A study of the Autism-Spectrum Quotient (AQ) and the NEO-PI-R.Personal Individ Differ 2006;41:873-83.

(21) Sizoo B, Brink W, Eenige MG,Gaag RJ.Personality characteristics of adults with autism spectrum disorders or attention deficit hyperactivity disorder with and without substance use disorders. JNervMent Dis, 2009;197(6):450-54.

(22) Vuijk R, de Nijs PFA, Vitale SG, Simons-Sprong M,Hengeveld MW. Persoonlijkheidsaspectenbijvolwassenen met autismespectrumstoornissengemeten met de 'Temperament and Character Inventory' (TCI). Tijdschrpsychiatr, 2012;54 (8):699-707.

(23) Ozonoff S, García N, Clark E,Lainhart J. MMPI-2 Personality profiles of high-functioning adult with autism spectrum disorders. Assessment 2005;1 (12):86-95.

(24) Butcher JN, Grant W, Graham JR, Tellegen A,Kaemmer B.Inventario Multifásico de Personalidad de Minnesota - 2, Madrid: TEA ediciones, 1989.

(25) Lord C, Rutter M, DiLavore PC, Risi S.Escala de Observación para el Diagnóstico de Autismo (ADOS), Madrid: TEA Ediciones, 2008.

(26) Rutter M, Le Couteur A, Lord C.Entrevista para el Diagnóstico del Autismo revisada (ADI-R); Madrid: TEAEdiciones, 2006.

(27) Wechsler D.Escala de Inteligencia para Adultos-III. Madrid: TEA Ediciones, 1999.

(28) Cohen J.A power primer. Psychol Bull, 1992;112:155-9.

(29) Nichols DS.Claves para la evaluación con el MMPI-2, Madrid: TEA ediciones, 2002.

(30) Allen D, Evans C, Hider A, Hawkins S, Peckett S, Morgan H. Offending Behaviour in 
Adults with Asperger Syndrome. JAutism DevDisord, 2008;38:748-58.

(31) Ghaziuddin M.Mental health aspects of Autism and Asperger Syndrome, London: Jessica Kingsley Publishers, 2005.

(32) Gillberg C,Billstedt E.Autism and Asperger syndrome: coexistence with other clinical disorders, ActaPsychiatrScand, 2000;102:321-30.

(33) Stewart ME, Barnard L, Pearson J, O'Brien G.Presentation of depression in autism and Asperger syndrome: A review. Autism 2006;10:103-16.

(34) Whitehouse AJ, Durkin K, Jaquet E,Ziatas K.Friendship, loneliness and depression in adolescents with Asperger's Syndrome. J Adolesc, 2008;32:309-22.

(35) American Psychiatric Association. DSM-5 Development. http://www.dsm5.org. 